


$$
\text { Vol. } 24, \mathrm{~N}^{\circ} 3
$$

Julio - Septiembre 2016

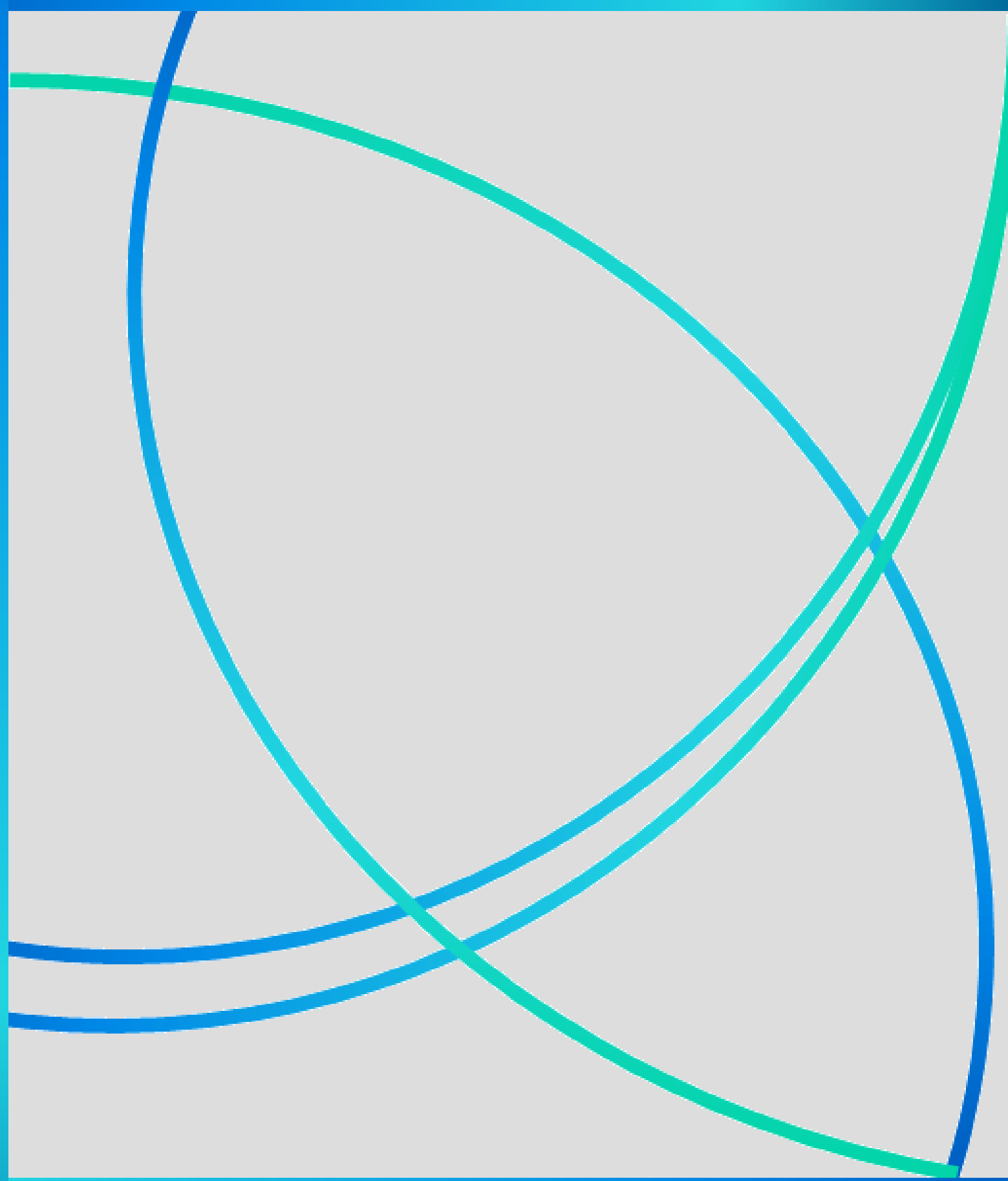

Esta publicación científica en formato digital es continuidad de la revista impresa Depósito Legal: pp $199302 Z U 47$ ISSN: 1315-2076
An International Refereed Scientific Journal of the Facultad Experimental de Ciencias at the Universidad del Zulia 
CIENCIA 24(3), 142-152, 2016

Maracaibo, Venezuela

\title{
Especiación de metales en sedimentos del río Cuchivero, Venezuela
}

\author{
Aristide Márquez, ${ }^{1}$, Gregorio Martínez', Julio Figuera², William \\ Senior ${ }^{1}$, Antonio Benítez ${ }^{1}$, Ángel González
}

${ }^{1}$ Departamento de Oceanografía, Instituto Oceanográfico de Venezuela, Universidad de Oriente. ${ }^{2}$ Departamento de Química, Universidad de Oriente, Núcleo de Sucre, Venezuela. 3Departamento de Recursos Acuáticos, Instituto Limnológico, Universidad de Oriente, Núcleo Bolívar, Venezuela

Recibido: 24-11-15 Aceptado: 29-07-16

\section{Resumen}

La concentración total y las formas químicas de los metales pesados se determinaron en sedimentos superficiales del río Cuchivero, Venezuela, empleando extracciones secuenciales y, espectrofotometría de absorción atómica con llama. Se utilizaron índices de contaminación, para estudiar la distribución, contaminación, y riesgo ambiental en los sedimentos. La concentración total de los metales osciló entre, 394-457 mgkg-1 Fe, 46,8774,82 Mn, 6,45-11,92 Zn, 1,70-5,75 Ni, 0,39-3,7 Cu, 1,25-3,63 Cr, 0,37-1,82 Co, o,10-0,30 Cd y <Ld-o,20 mgkg-1 Pb. Los metales se asociaron fuertemente a la fracción residual de los minerales (70 a 90\%), carbonatos y a los oxihidróxidos de hierro. Manganeso y cadmio, se asociaron principalmente a la fracción intercambiable (60-70\%). Los índices indican que la fuente de los metales es principalmente la roca madre, y que no existe enriquecimiento por metal, ni contaminación. Por otra el parte, riesgo de alteración ambiental es bajo para $\mathrm{Fe}$ y $\mathrm{Co}$, alto para $\mathrm{Zn}$ y Ni, medio para $\mathrm{Cu}$ y $\mathrm{Cr}$, y muy alto para $\mathrm{Mn}$, $\mathrm{Cd}$ y $\mathrm{Pb}$.

Palabras clave: Especiación, metales, sedimentos, ríos tropicales, ambiental.

\section{Speciation of heavy metals in sediments of the Cuchivero River, Venezuela}

\begin{abstract}
The total concentration and the chemical forms of heavy metals were determined in superficial sediments of the Cuchivero river, Venezuela, using sequential extraction and atomic absorption spectrophotometry with flame. Pollution indices were used to study the distribution, pollution and environmental risk in the sediments. Total values ranged from, 394-457 mgkg-1 Fe, 46,87-74,82 Mn, 6,45-11,92 Zn, 1,70-5,75 Ni, 0,39-3,7 Cu, 1,25-3,63 $\mathrm{Cr}, 0,37-1,82 \mathrm{Co}, 0,10-0,30 \mathrm{Cd}$ y $<\mathrm{Ld}-0,20 \mathrm{mgkg}-1 \mathrm{~Pb}$. The metals were found to be strongly associated with the residual fraction minerals (70 to 90\%), carbonates and iron oxihidroxides. Manganese and cadmium were associated mostly exchangeable fraction (60-70\%). The indices indicate that the source of metals is mainly bedrock; no metal enrichment and contamination exist. The risk of environmental disruption is low for $\mathrm{Fe}$ and $\mathrm{Co}$, high for $\mathrm{Zn}$ and $\mathrm{Ni}$, medium for $\mathrm{Cu}$ and $\mathrm{Cr}$, and very high for $\mathrm{Mn}, \mathrm{Cd}$ and $\mathrm{Pb}$.
\end{abstract}

Keywords: speciation, metals, sediments, tropical rivers, environmental.

*Autor para la correspondencia: aristd@gmail.com 


\section{Introducción}

Los metales pesados tienen un gran significado como indicadores de la calidad ecológica de las fuentes de agua, debido a su toxicidad y especialmente a su comportamiento bioacumulativo. Es por ello que son considerados como punto de partida en muchas investigaciones a nivel ambiental. Los ríos de la Guayana venezolana presentan sedimentos sumamente lixiviados, ricos en oxihidróxidos de hierro y aluminio, lo que ha permitido considerar la región como un frágil ecosistema, susceptible a cambios bruscos por la posible intervención desmedida e irracional del hombre (1). El río Cuchivero se encuentra en la provincia de Cuchivero está situada en la parte occidental del escudo Guayanés Venezolano, con rocas plutónicas de edad proterozoicas, que afloran en las cuencas de los ríos Paragua y Cuchivero, proporcionado a toda la región condiciones geológicas especiales (2).La pequeña minería de oro $y$ diamante en la cuenca de los ríos Cuchivero, Caroní y Cuyuní ha producido daños irreversibles en los ecosistemas terrestres y fluviales localizados en algunos puntos de estas cuencas (3).

Para comprender el comportamiento de los metales en el medio ambiente, es necesario obtener información sobre la especiación química de estos elementos, es decir, determinar las formas orgánicas e inorgánicas específicas que forman parte de la concentración total del elemento en solución, al igual que las contenidas en sedimentos (4). Los sedimentos pueden actuar como portadores y posible fuente de contaminación porque los metales adsorbidos en ellos pueden ser liberados a la columna de agua por cambios en las condiciones ambientales tales como $\mathrm{pH}$, potencial redox, oxígeno disuelto o la presencia de quelatos orgánicos. En consecuencia, el estudio en la fracción biodisponible o móvil de metales ligados a sedimentos es más importante que la concentración total del metal en las corrientes fluviales (5). La falta implementación de logísticas adecuadas ha impedido en muchos casos el acceso para estudiar los grandes ríos de la región de Guayana, en la cual se encuentra el río Cuchivero, esto ha originado que los estudios geoquímicos sobre todo de metales pesados en este ecosistema sean escasos. En vista de lo expuesto, está investigación tuvo como objetivo principal determinar las diferentes fracciones de metales, $\mathrm{Fe}, \mathrm{Mn}, \mathrm{Pb}, \mathrm{Cd}, \mathrm{Zn}$, $\mathrm{Cu}, \mathrm{Ni}, \mathrm{Co}, \mathrm{Cr}$, asociadas a la matriz sedimentaria.

\section{Materiales y métodos}

El área de estudio estuvo ubicada en el río Cuchivero, el cual está localizado en el sur de Venezuela, en el estado Bolívar (Fig.1), nace en la vertiente noroeste de la altiplanicie de Icutú, en la serranía de Guamapí de la sierra de Maigualida. Drena una cuenca de aproximadamente $16.000 \mathrm{~km}^{2}$ de extensión, tiene una longitud de 305 kilómetros y desemboca en el río Orinoco en la ciudad de Caicara del Orinoco. En su recorrido recibe, por el oeste o margen izquierdo, al río Guaniamo, su principal afluente, y por el este, al Sariapo (6). Se utilizó como plataforma de trabajo, un bote peñero de 6 metros de largo para la recolección de los sedimentos superficiales, en diez estaciones de muestreo aguas abajo a lo largo del río Cuchivero, desde la localidad de Puerto Matú en la cual se encuentran las minas de diamantes (estación 1), hasta su desembocadura en el río Orinoco (estación 10), en un trayecto de $55 \mathrm{~km}$. Del grupo de estaciones muestreadas, se recolectó una muestra dentro del río Guaniamo (estación 5). Para la ubicación geográfica de las estaciones se utilizó un GPS marca Garmin 12XL (EUA). Las muestras se recolectaron utilizando una draga tipo 
Diez Laffont de o,o2m ${ }^{2}$ de área (EUA). Con el apoyo de una paleta plástica las muestras se colocaron en frascos de polipropileno de $250 \mathrm{~mL}$ de capacidad previamente rotulados y lavados con una solución ácida ( $\mathrm{HCl} 1 \mathrm{molL}^{-1}$; Merck) y agua desionizada, calidad Nanopure de conductividad de $18{\mathrm{M} \Omega \mathrm{cm}^{-1} \text {. Las }}$ muestras luego se preservaron bajo congelación a $\quad-20 \quad{ }^{\circ} \mathrm{C}$ hasta $\mathrm{su}$ procesamiento.

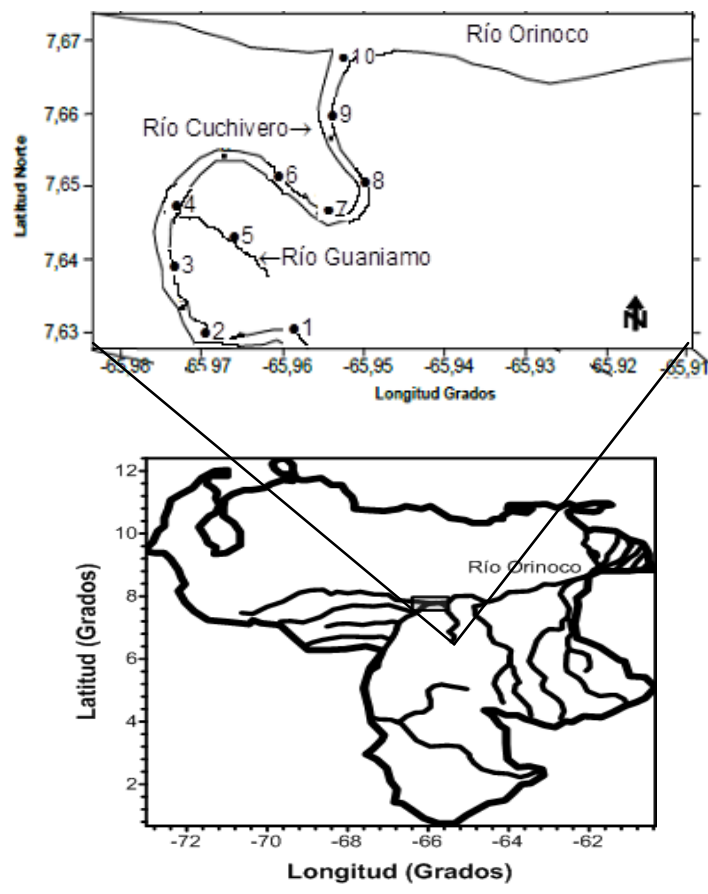

Figura 1. Zona de estudio mostrando los sitios de recolección de las muestras en el río Cuchivero, Venezuela

Para el análisis granulométrico, los sedimentos fueron secados a $80^{\circ} \mathrm{C}$ en una estufa p SELECTA (EUA) y luego Pasados través de tamices de 4,00; 2,00; 1,00; 0,85; 0,50; 0,25 y 0,063 mm. Para los análisis, los sedimentos preservados fueron descongelados y secados a $60^{\circ} \mathrm{C}$ en una estufa hasta obtener un peso constante, luego se tamizaron para obtener la fracción menor a $2 \mathrm{~mm}$ y se pulverizaron en un mortero de porcelana, para luego ser almacenados en envases de polietileno previamente lavados hasta los análisis $(7,4)$. Todos los análisis fueron realizados en el Laboratorio de Química Marina del Departamento de Oceanografía, Instituto Oceanográfico de Venezuela.

La materia orgánica total fue calculada por diferencia de peso a través del método de calcinación a $540^{\circ} \mathrm{C}$ descrito en (8). Para ello se utilizó una mufla Marca JELRUS y una balanza analítica Marca Denver Instrument M-10 (USA) con precisión de o,ooo1g. Los carbonatos se determinaron por titulación volumétrica con $\mathrm{NaOH}$ 0,50 molL $^{-1}$ (9). Para ello, se pesó 0,50 g de sedimentos previamente calcinados, se añadió $15 \mathrm{~mL}$ de $\mathrm{HCl}$ o,1 $\mathrm{molL}^{-1} \mathrm{y}$ se calentó hasta ebullición. Las muestras se filtraron para eliminar la cantidad de sedimento que obstaculizaba el cambio de color del indicador en el punto final de la titulación y se valoró con $\mathrm{NaOH}$ 0,29 molL ${ }^{-1}$, usando como indicador azul de bromofenol. Los análisis de materia orgánica, y carbonatos se realizaron para determinar las asociaciones estadísticas de los metales con estas variables.

La especiación de metales se realizó utilizando el método descrito por Tessier y col., 1979 (10), el cual describe extracciones secuenciales en cuatro etapas. Para extraer la fracción $(\mathrm{F} 1)$ de los metales intercambiables que se encuentran adsorbidos a superficie del sedimento, dos (2) gramos de sedimento seco fue extraído por dos (2) horas a temperatura ambiente con $15 \mathrm{~mL}$ de un buffer de acetato de amonio $1 \mathrm{molL}^{-1} \mathrm{a} \mathrm{pH}$ 7. Segunda etapa (F2). El residuo de la fracción 1, se colocó en un enlermeyer de $125 \mathrm{~mL}$, se agregó $15 \mathrm{~mL}$ de ácido acético al $25 \% \mathrm{v} / \mathrm{v}$ y se extrajo a temperatura ambiente durante cuatro (4) horas, los metales asociados a los iones carbonato. Tercera etapa $\left(\mathrm{F}_{3}\right)$. Al residuo de la fracción 2, se le agregan $10 \mathrm{~mL}$ de ácido acético al $25 \% \mathrm{v} / \mathrm{v}$ y $10 \mathrm{~mL}$ de peróxido de 
hidrógeno al $30 \% \mathrm{v} / \mathrm{v}$. La solución fue filtrada, $\mathrm{y}$ se ajustó el $\mathrm{pH}$ a 2, posteriormente se calentó en una plancha de calentamiento durante dos (2) horas a $85^{\circ} \mathrm{C}$. El residuo se trató con $5 \mathrm{~mL}$ de acetato de amonio 3,2 $\mathrm{molL}^{-1}$ en ácido nítrico 20\% v/v. En esta etapa, se extrajeron los metales adsorbidos a los óxidos e hidróxidos de hierro y manganeso y presentes en la materia orgánica. Cuarta etapa (F4). El residuo del paso anterior, se trató con $10 \mathrm{~mL}$ de ácido nítrico, ácido clorhídrico y ácido perclórico en una relación 3:2:1v/v, utilizando un digestor de Microondas Microdigest 401 de Prolabo (Francia), a $110^{\circ} \mathrm{C}$, por 30 minutos para mineralizar el sedimento, luego se dejó enfriar y se filtró. Cada fracción fue aforada en balones de $25 \mathrm{~mL}$. Las concentraciones de los metales estudiados se determinaron utilizando un espectrofotómetro de absorción atómico marca Perkin Elmer 3110 con llama de aire acetileno y corrector de fondo de deuterio.

Para determinar el enriquecimiento del sedimento en relación al metal, el grado de contaminación e incluso la toxicidad del sedimento, se utilizaron los índices de geoacumulación (Igeo), factor de enriquecimiento (FE), índice potencial de contaminación (CP) y el índice de capacidad de carga (ICC). Se utilizó la formula Igeo $=\log _{2}[\mathrm{Cn} / 1,5 B n]$, donde $\mathrm{C}$ es la concentración para un metal $n$ en el sedimento y $\mathrm{B} n$ es el valor de fondo del metal en la corteza terrestre del ecosistema bajo estudio. El factor 1,5 es utilizado debido a las posibles variaciones en los datos de fondo originados por variaciones litológicas (11). En este estudio se utilizó la fracción $<63 \mu \mathrm{m}$ debido a que esta fracción ha sido utilizada en previas investigaciones (12, 13,10). Considerando que se desconocen los valores de fondo de cada metal en la corteza terrestre de la zona bajo estudio, se utilizaron los valores promedios para la corteza terrestre propuestos por Turekiank y Wedepohl, 1961 (14). La discusión de los resultados se realizó en función de los argumentos señalados por Müller, 1979 (15).

Debido a que algunos autores han criticado el índice de geoacumulación para indicar contaminación (16), se ha propuesto que los valores obtenidos en las concentraciones, sean normalizados. Algunos elementos como $\mathrm{Fe}, \mathrm{Al}, \mathrm{COT}$ y tamaño del grano han sido utilizados para la normalización (10). En esta investigación se ha utilizado como factor de normalización el hierro. El hierro fue utilizado porque es una de las mayores fases adsorbentes de metales y es un trazador cuasi conservativo de la movilidad del metal en las fases dentro de los sedimentos fluviales (17) y su geoquímica es similar a la de muchos metales trazas en medios ambientes óxidos y anóxicos (10). Para esta investigación el factor de enriquecimiento fue calculado utilizando la expresión $\mathrm{FE}=$ (Metal/Fe) sedimento/(metal/Fe)Bn, donde $B n$ son los valores de fondo reportados por Turekiank y Wedepohl, 1961 (14) para la corteza terrestre. Las interpretaciones fueron hechas a partir de las sugerencias realizadas por Taylor, 1964 (18). Para determinar el origen del metal se utilizó las escalas de los FE (19).El índice potencial de contaminación (CP) fue obtenido a partir de la expresión, $\mathrm{CP}=[$ metal $]$ máx / [Metal $] \mathrm{Bn}$, en la cual, [metal] es la concentración máxima del metal en sedimento (20). El Índice de carga contaminante (ICC), fue evaluado para toda la zona de acuerdo a, $\mathrm{ICC}==\left(\mathrm{CP}_{1}+\mathrm{CP}_{2}+\right.$ $\left.\mathrm{CP}_{3} \ldots \ldots+\ldots \ldots . \mathrm{CPn}\right)^{1 / \mathrm{N}} ; \quad$ donde $\mathrm{CP}=[$ metal $] / \mathrm{B} n ;$ y $[$ metal $]=$ concentración del metal en el sedimento y $\mathrm{N}=$ número de metales evaluados. Para esta investigación $\mathrm{N}=9$ (21).

Los criterios utilizados para interpretar estos índices fueron: Si, Igeo 
$=\mathrm{O}=$ no contaminado, $[\mathrm{O}-1]=$ no contaminado a moderadamente contaminado; $[1-2]=$ moderadamente contaminado; $[2-3]=$ de moderado a fuertemente contaminado; [3-4] = fuertemente contaminado; $[4-5]=\mathrm{de}$ fuerte a extremadamente contaminado; [5-6] = extremadamente contaminado (15). $\mathrm{FE}<1$ no enriquecido; $\mathrm{FE}=1-5$; enriquecimiento moderado; $\mathrm{FE}=5-10$ de moderado a severamente enriquecido; FE $=10-25$ severamente enriquecido, $\mathrm{FE}>$ 25-50 muy severamente enriquecido; FE $>25-50=$ extremadamente enriquecido (18). $\mathrm{CP}>3$ contaminación severa . $\mathrm{Si} \mathrm{FE}=$ $0-1=$ origen de la roca madre; $\mathrm{FE}=1-10-$ 500 otra fuente adicional de la roca madre; $\quad \mathrm{FE}>500=$ contaminación antropogénica (19). Icc $<8=$ bajo grado de contaminación; $8 \leq$ Icc $<16$ moderado grado de contaminación; $16 \leq$ Icc $<32$, considerable grado de contaminación; Icc $\geq 32$, muy alto grado de contaminación (21).

Para evaluar el riesgo ambiental en el sedimento se empleó el Código de Evaluación de Riesgo (RAC), el cual clasifica el riesgo de acuerdo al porcentaje del elemento que se encuentran en el sedimento como intercambiable o carbonato $\left.(\mathrm{F} 1+\mathrm{F} 2) / \mathrm{FT}^{*} 100\right)$, en donde $\mathrm{F} 1$ es la fracción 1; F2 es la fracción 2 y FT es la concentración. Valores de este factor menores a 1 no representa riesgo, entre 110 el riesgo es bajo, 11-30 es medio, 31-50 es alto y 51-71 es muy alto (22).

La precisión de los análisis de MOT y $\mathrm{CaCO}_{3}$ fue chequeada tomando una muestra de sedimento al azar y analizando cuatro réplicas del mismo. Los bajos valores de la desviación estándar y el coeficiente de variación evidencian buena reproducibilidad de los análisis. Por otra parte, la calidad analítica de los datos obtenidos del análisis de los metales, se chequeó con un patrón de sedimento certificado por la Enviromental Resource Associates: Catálogo número 540, lote 237 (PriorityPollutn/CPL. Soil). Los porcentajes de extracción de metales en las muestras estudiadas fueron bastantes representativos, tal como lo demuestran la desviaciones estándar bastante bajas para las cinco (5) réplicas analizadas y la comparación de los promedios obtenidos con el rango, aceptable y el valor del patrón certificado (Tabla 1). Toda el agua utilizada tanto en la preparación de reactivos, curvas de calibración y blancos de reactivos fue agua desionizada altamente pura (agua calidad NANOPURE de conductividad de 18 $\left.\Omega \mathrm{cm}^{-1}\right)$. Esto fue obtenido con un sistema NANOPURE UV, Marca Barnstead. Para determinar asociaciones entre parámetros se realizó análisis de correlación de Pearson utilizando el paquete STATGRAPHICS PLUS 5.1.

Tabla 1. Precisión del método de extracción de $\mathrm{MOT}, \mathrm{CaCO}_{3}$, y de metales pesados (sedimento de referencia, patrón Lot. $\mathrm{N}^{\mathrm{O}}$ 237.Cat. $\mathrm{N}^{\mathrm{o}} 540$ para sedimentos del sistema del río Cuchivero. Venezuela

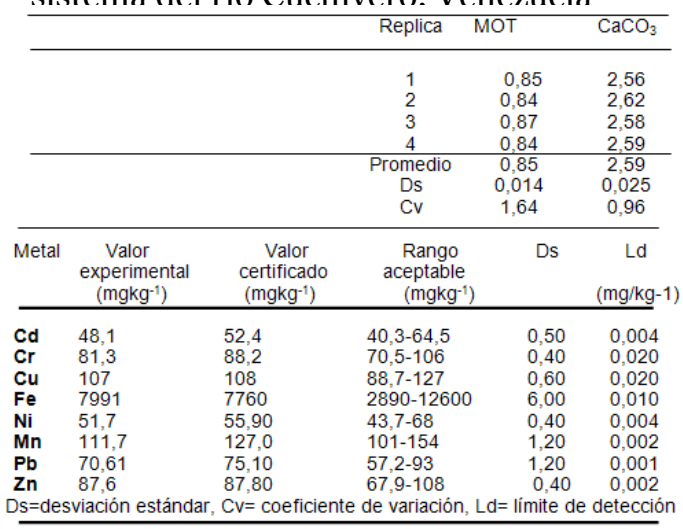

\section{Resultados y discusión}

La granulometría de los sedimentos del río Cuchivero estuvo tipificada por un predominio de las arenas de tipo media y fina con bajos porcentajes de lodos. Las arenas medias oscilaron entre $38-45 \%$, las finas entre 34 - $40 \%$ y loa lodos entre 15-27\%. En el río Guaniamo estos valores 
fueron $45 \%$, 40\% y $15 \%$ respectivamente (figura 2A). Los altos porcentajes de arenas evidencian un lavado constante de los sedimentos debido a la alta energía de las aguas de este sistema fluvial. La cuenca del Cuchivero se encuentra situada en la parte occidental del Escudo Guayanes, y discurre por la provincia

A

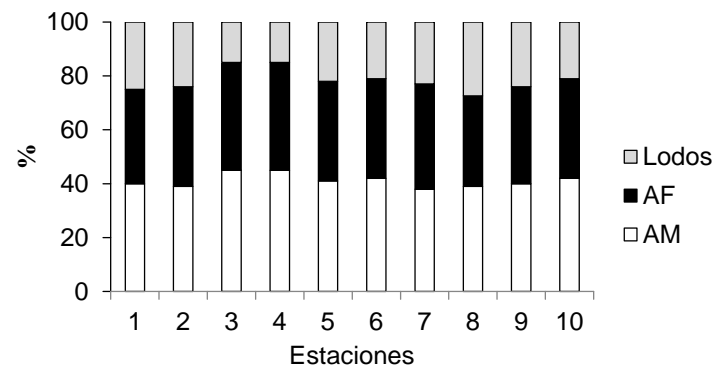

C

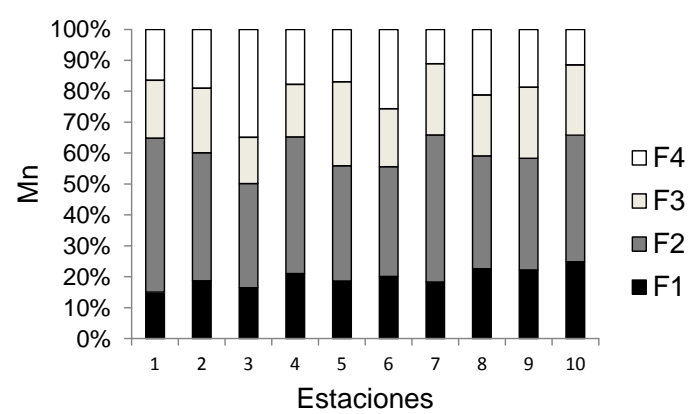

$\mathrm{E}$

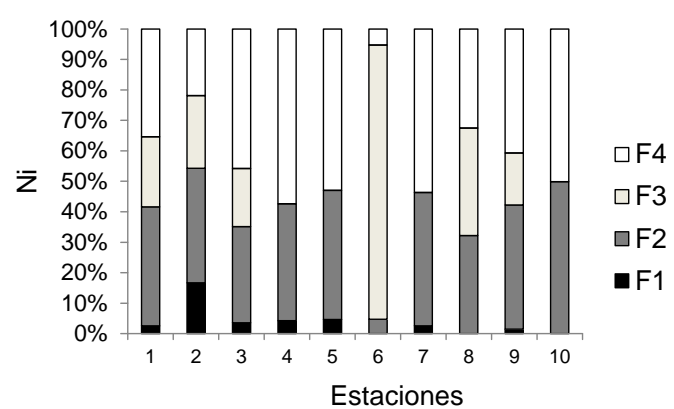

estructural que recibe su mismo nombre, la cual expone extensamente rocas plutónicas, epi-meso y meso-zonales, con vestigios mucho menos voluminosos de rocas meta-volcánicas de composición predominantemente silíceas y metasedimentarias de disposición clásticas (COLVEÉ et al.1990).

B

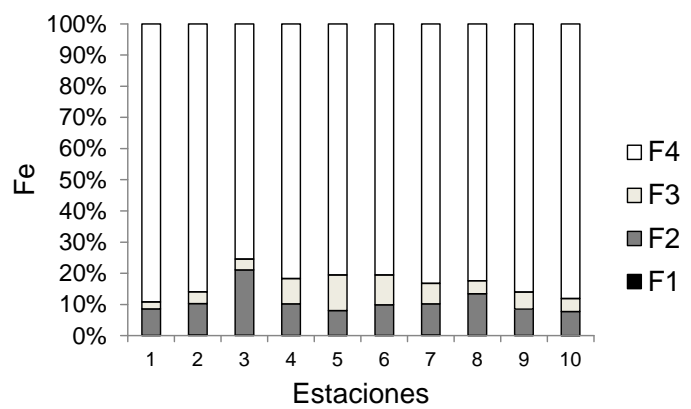

D

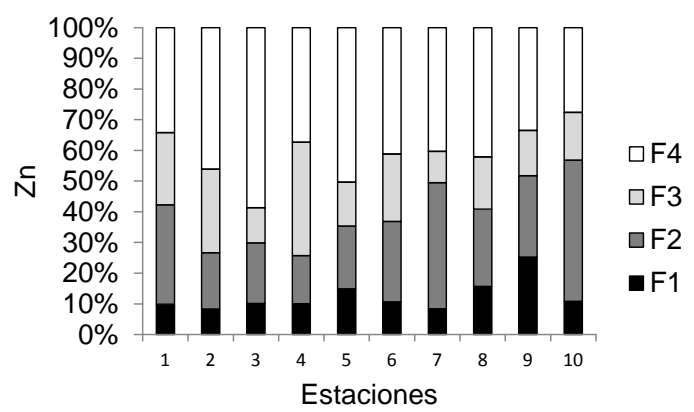

$\mathrm{F}$

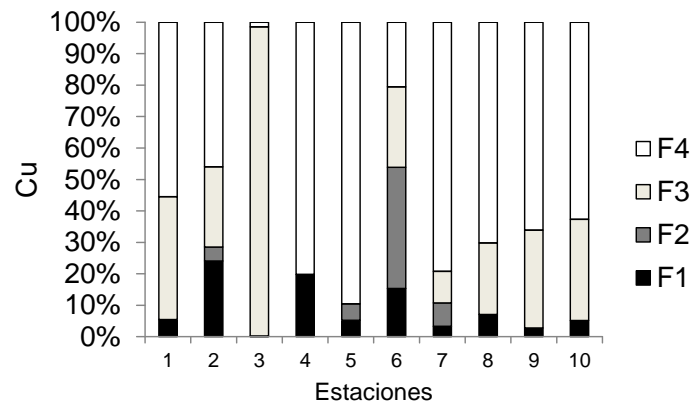

Figura 2. Tamaño de la partícula (2A) y concentraciones de metales pesados en las diferentes fracciones del sedimento del río Cuchivero, Venezuela 
Las concentración de los diferentes metales en los sedimentos del sistema del río Cuchivero oscilaron en un rango de 394-457 mgkg-1 $^{-1} \mathrm{Fe}, 46,87-74,82 \mathrm{Mn}$, 6,45-11,92 Zn, 1,70-5,75 Ni, 0,39-3,7 Cu, 1,25-3,63 Cr, 0,37-1,82 Co, 0,10-0,30 Cd y <Ld-0,20 mgkg $^{-1} \quad \mathrm{~Pb}$. Los valores promedios $\left(\mathrm{mgkg}^{-1}\right)$ fueron 423 de $\mathrm{Fe}$; 64,19 de Mn; 9,02 de Zn; 3,87 de Ni; 2,25 de $\mathrm{Cu} ; 2,07$ de $\mathrm{Cr} ; 1,16$ de $\mathrm{Co} ; 0,21$ de $\mathrm{Cd}$ y 0,07 mgkg-1 de $^{-1} \mathrm{~Pb}$ respectivamente (Tabla 2).

Tabla 2. Concentraciones de metales (mgkg-1) en sedimentos del sistema del río Cuchivero, Venezuela

\begin{tabular}{|c|c|c|c|c|c|c|c|c|c|c|}
\hline & & $\mathrm{Fe}$ & & $\mathrm{Zn}$ & $\mathrm{Ni}$ & $\mathrm{Cu}$ & $\mathrm{Cr}$ & $\mathrm{Co}_{0}$ & $2 d$ & $\mathrm{~Pb}$ \\
\hline & & 436 & 64,08 & 7,92 & 66 & 3,28 & 1,52 &, 03 & 3 & $-\mathrm{T}$ \\
\hline & 2 & 403 & 65,94 & $7,8_{3}$ & 5,75 & 3,7 & 2,12 & 0,85 & 0,29 & Lach \\
\hline & 3 & 457 & 69,53 & 0,07 & $4,5^{\circ}$ & 2,6 & 14 & 0,37 & 14 & 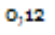 \\
\hline & 4 & 408 & 68,63 & 9,24 & 11 & 1,01 & 1,84 & 1,3 & & 0,18 \\
\hline & 5 & $45^{6}$ & 70,33 & 9,11 & 4,53 & 2,69 & 2,33 & 1,73 & 0,20 & 0,15 \\
\hline & 6 & 412 & 74,82 & 11,92 & 1,70 & 0,39 & 55 & 19 &, 29 & $<\mathrm{L}$ \\
\hline & 7 & 428 & 58,34 & 10,46 & 2,70 & 1,49 & 25 & 1,11 & 11 & 0,2 \\
\hline & 8 & $43^{\circ}$ & 63,4 & 6,95 & 3,04 & 1,41 & 72 & 0,73 & 26 & $<\mathrm{LC}$ \\
\hline & 9 & 394 & 59,96 & 6,45 & 5,26 & 3,22 & 2,55 &, 43 & 19 & 0,07 \\
\hline & 10 & 404 & 46,87 & 10,25 & 3,43 & 2,73 & 3,63 & 1,82 & 0,17 & $<\mathrm{Ld}$ \\
\hline & & 394 & 46,87 & 6,45 & 1,70 & 0,39 & 1,25 & 9,37 & 0,10 & 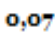 \\
\hline$\theta$ & & 457 & $74,8=$ & 11,92 & 5,75 & 3,70 & 3,63 & $1,8=$ & 0,30 & $0, z$ \\
\hline Prom & & $4=3$ & 64,19 & 9,02 & 3,87 & 2,25 & 2,07 & 1,16 & 0,21 & 0,1 \\
\hline
\end{tabular}

Min=mínimo, Max= máximo, Prom=promedio

Los valores para la muestra recolectada en el río Guaniamo fueron 456; 70,33; 9,11; 4,53; 2,69; 2,33; 1,73; 0,20 y 0,15 mgkg-1 $^{-1}$ para Fe, Mn, Zn, Ni, $\mathrm{Cu}, \mathrm{Cr}, \mathrm{Co}, \mathrm{Cd} \mathrm{y} \mathrm{Pb}$ respectivamente. $\mathrm{La}$ especiación mostró que el hierro se distribuyó entre $0,14-0,17 \mathrm{mgkg}^{-1}$ en la fracción $\mathrm{F} 1 ; 31,19-95,61 \mathrm{mgkg}^{-1}$ en la fracción F2; 9,81-39-32 mgkg-1 $^{-1}$ en F3 y 331,59-388,41 mgkg $^{-1}$ en F4, evidenciándose una preferencia superior al $90 \%$ hacia la fracción residual (Fig.2B). La distribución por fracciones para hierro en promedio fue $\mathrm{F}_{4}$ $>F_{2}>F_{3}>F_{1}$. Para manganeso los valores variaron entre 9,56-15,05 $\mathrm{mgkg}^{-1} \mathrm{~F}$; 19,18-31,92 mgkg-1 $^{-1}$ F2; 16,22 - 14,03 $\mathrm{mgkg}^{-1} \mathrm{~F} 3 ;$ 5,37-23,93 mgkg-1 $^{-1} 4$ con una distribución $\quad \mathrm{F} 2>\mathrm{F}_{1} \approx \mathrm{F}_{3} \approx \mathrm{F}_{4}$, evidenciándose una mayor asociación del metal entre 50 y $65 \%$ del total en las fracciones F2 y F1 (Fig.2C). El zinc varió entre $0,65-1,63$ mgkg-1 $^{-1}$ F1: 1,44-4,72

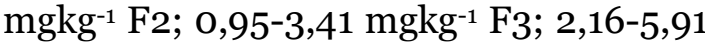
$\mathrm{mgkg}^{-1} \mathrm{~F} 4$, mostrando una distribución heterogénea con gradiente creciente $\mathrm{F}_{4}>\mathrm{F}_{2}>\mathrm{F}_{3}>\mathrm{F}_{1}$, apreciándose una mayor tendencia a estar en la fracción residual, sin embargo la suma de las fracciones $\mathrm{F} 1+\mathrm{F}_{2}+\mathrm{F}_{3}$ alcanzan en conjunto valores entre el 50-70\% (Fig. 2D).Las fracciones de níquel oscilaron entre no detectado0,96F1; 0,08-2,16 F2; <Ld-1,53 F3; 0,092,14 $\mathrm{mgkg}^{-1} \mathrm{~F} 4$, con una secuencia $\mathrm{F}_{4}>\mathrm{F}_{2}>\mathrm{F}_{3}>\mathrm{F} 1$, mostrando al igual que el zinc una tendencia hacia la fase residual.

La concentración de cobre estuvo muy alternada entre las estaciones, y fluctuó entre $0,05-1,13 \mathrm{~F} 1$; no detectado0,21 F2; no detectado-1,28 F3; 0,08-2,16 F4. En promedio la tendencia de la distribución fue $\mathrm{F}_{4}>\mathrm{F}_{3}>\mathrm{F}_{1}>\mathrm{F}_{2}$, con asociación la fracción residual. Sin embargo estaciones como la número tres, presentó casi el 100\% del metal asociado F3 (Fig.2F). Cromo varió entre <Ld-0,93 F1; 0,08-0,33 mgkg $^{-1}$ F2; 0,31-0,83 mgkg1 F3; 0,71-1,29 $\mathrm{mgkg}^{-1} \mathrm{~F} 4$, con valores superiores al $40 \%$ en la fracción residual (Fig.3A). La secuencia mostrada en la especiación fue $\mathrm{F}_{4}>\mathrm{F}_{3}>\mathrm{F}_{1}=\mathrm{F}_{2}$. $\mathrm{El}$ cobalto no fue detectado en la fracción $\mathrm{F} 1$ y la fracción F2 se determinó solo en las estaciones 1,4 y 8, donde los valores oscilaron entre no detectado-0,09 mgkg-1. Las concentraciones se repartieron entre F3 y F4 donde los valores fluctuaron entre 0,07-0,71 mgkg-1 $^{-1}$ 1 y o,09-1,02 $\mathrm{mgkg}^{-1} \mathrm{~F} 2$, representando entre ambos más del 90\% del metal, con una mayor tendencia hacia la fracción residual $\mathrm{F} 4$ (Fig.3B).La secuencia en la especiación fue $\mathrm{F}_{4}>\mathrm{F}_{3}>\mathrm{F} 2$. El valor de cadmio en $\mathrm{F} 1$ varió entre no detectado- $0,06 \mathrm{mg} / \mathrm{kg}$ y en F2 desde 0,05-0,21 $\mathrm{mgkg}^{-1}$ representando alrededor del $60 \%$ del metal respecto al 
total. La fracción $\mathrm{F}_{3}$ al igual que $\mathrm{F}_{4}$ osciló entre no detectado- $0,09 \mathrm{mgkg}^{-1}$ representando entre el 10 y $30 \%$. En la estación 8, el cadmio representó cerca del $90 \%$ del metal respecto al total y en la estación $4 \quad \mathrm{~F} 2$ el $100 \%$ del metal (Fig.3C).La secuencia de la especiación fue $\mathrm{F}_{2}>\mathrm{F}_{1}>\mathrm{F}_{3}=\mathrm{F}_{4}$, evidenciando que el $\mathrm{Cd}$ se encuentra en su mayoría en forma biodisponible.

A

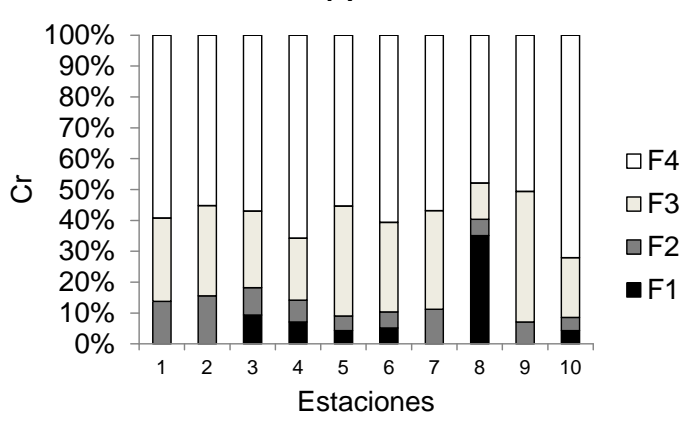

C

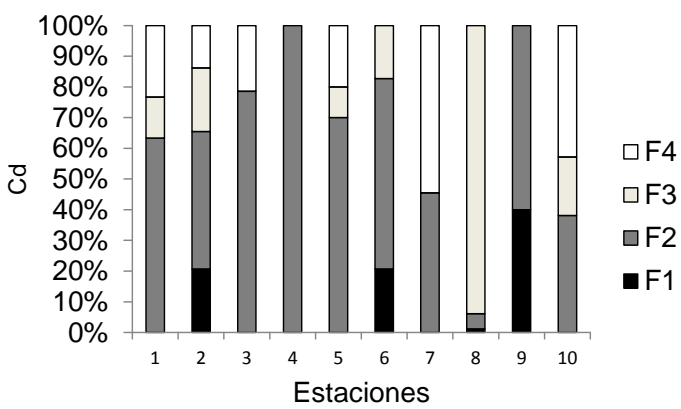

E

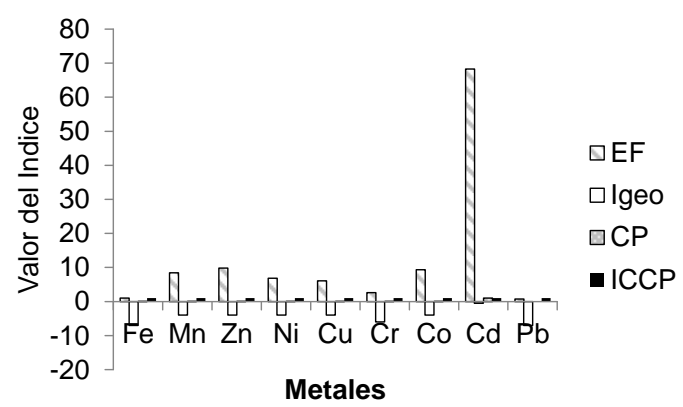

El plomo solo se detectó en las estaciones 3,4, 5 7,8 y 10 en las cuales las fracciones presentes fueron $\mathrm{F}_{3}$ con valores entre no detectado- $0,07 \mathrm{mgkg}^{-1}$. En las estaciones 3,4 y 7 la fracción residual representó el $100 \%$ respecto al total y en las estaciones 8 y 10 fue la fracción $\mathrm{F}_{3}$ que reflejó un comportamiento similar (Fig.3D).
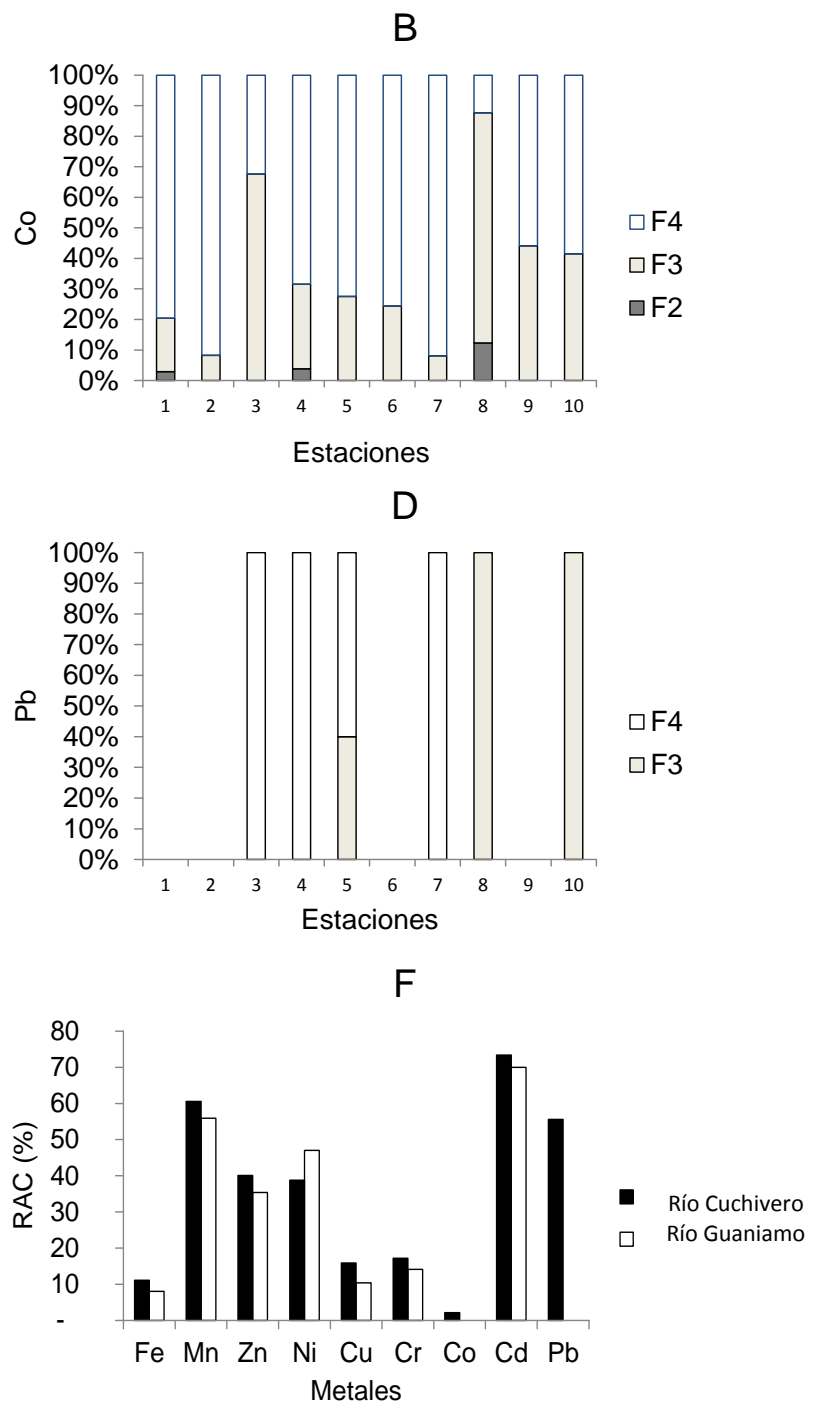

Figura 3. Concentraciones de metales pesados en las diferentes fracciones e índices de contaminación en sedimentos del río Cuchivero, Venezuela 
La secuencia de la especiación fue $\mathrm{F}_{3}=\mathrm{F} 4$. Para la estación cinco, la cual está dentro del río Guaniamo, la especiación mostró valores en la fracción $\mathrm{F} 1$ de: 0,45 ; 13,1; 1,36. 0,21, 0,14; 0,13 mgkg-1 $^{-1}$ para Fe, $\mathrm{Mn}, \mathrm{Zn}, \mathrm{Ni}, \mathrm{Cu}, \mathrm{Cr}$ respectivamente y no detectado para $\mathrm{Co}, \mathrm{Cd}$ y $\mathrm{Pb}$. Para este mismo orden de representación de los metales, los valores de $\mathrm{F} 2$ variaron en 36,$06 ; 26,22 ; 1,86 ; 1,92,0,14 ; 0,13$ mgkg 1, no detectado; 0,14 mgkg-1 $^{-1}$ y no detectado. Para F3 los valores fueron, 52,24; 19,10; 1,30; <Ld; 0,37; 0,43; 0,02 y $0,06 \mathrm{mgkg}^{-1}$ para $\mathrm{Fe}, \mathrm{Mn}, \mathrm{Zn}, \mathrm{Ni}$ y $\mathrm{Cu}$, $\mathrm{Cr}, \mathrm{Co}, \mathrm{Cd} \mathrm{y} \mathrm{Pb}$ respectivamente. Por ultimo para la fracción $\mathrm{F}_{4}$ las concentraciones fueron de $367 ; 11,91$; 4,$59 ; 2,40,2,41 ; 1,21 ; 1,13,0,04$ y 0,09 mgkg-1 $^{-1}$ respectivamente. Las diferentes secuencias de especiación para los metales en el río Guaniamo fueron $\mathrm{F}_{4}>\mathrm{F}_{3}>\mathrm{F}_{2}>\mathrm{F}_{1}$ para $\mathrm{Fe} ; \mathrm{F}_{2}>\mathrm{F}_{1}>\mathrm{F}_{3}>\mathrm{F}_{4}$ para $\mathrm{Mn} ; \quad \mathrm{F} 4>\mathrm{F} 2>\mathrm{F}_{1}=\mathrm{F}_{3}$ para $\mathrm{Zn}$; $\mathrm{F}_{4}>\mathrm{F}_{2}>\mathrm{F}_{1}>\mathrm{F}_{3}$ para $\mathrm{Ni} ; \mathrm{F}_{4}>\mathrm{F}_{1}=\mathrm{F}_{2}>\mathrm{F}_{3}$ para $\mathrm{Cu}, \quad \mathrm{F}_{4}>\mathrm{F}_{3}>\mathrm{F}_{1}=\mathrm{F}_{2}$ para $\mathrm{Cr}$; $\mathrm{F}_{3}>\mathrm{F}_{4}>\mathrm{F}_{1}=\mathrm{F}_{2}$ para $\mathrm{Co}_{2} \mathrm{~F}_{2}>\mathrm{F}_{4}>\mathrm{F}_{3}>\mathrm{F}_{1}$ para $\mathrm{Cd}$ y $\mathrm{F}_{4}>\mathrm{F}_{3}$ para $\mathrm{Pb}$. Estas secuencias observadas tanto en los sedimentos del río Cuchivero, así como en el Guaniamo, reflejan la complejidad de la mineralogía prevaleciente en sus cuencas y evidencian que aunque la mayoría de los metales están presentes en la fase residual en su mayoría, algunos están presentes en las fracciones más biodisponibles como F1 y F2, lo cual indica que estos metales pueden estar disponibles para la biota.

Las correlaciones mostraron asociaciones entre la materia orgánica con los lodos, sin embargo, solo se apreciaron correlación positiva entre manganeso y cadmio con los lodos ( $\mathrm{r}=$ $0,63, \mathrm{r}=0,60$,). Otros metales como $\mathrm{Zn}$, $\mathrm{Ni}, \mathrm{Pb}$ y $\mathrm{Fe}(\mathrm{r}=0,72, \mathrm{r}=0,56, \mathrm{r}=0,52)$ están asociados con los carbonatos. $\mathrm{Cu}$, Co y Cr están asociados con el hierro $(\mathrm{r}=$ $0,56, r=0,52, r=0,68)$. Esto corrobora lo apreciado durante los diferentes pasos empleados en la especiación, lo cual confirma que, estos metales se encuentran presentes en los carbonatos, asociados a óxidos, preferiblemente a los de hierro, y en forma residual formando parte de la estructura intermolecular de los diferentes minerales. Por otra parte la correlación observada entre $\mathrm{Mn}$ y $\mathrm{Cd}$ con los lodos, evidencia que estos metales se encuentran adsorbidos por fuerzas intermoleculares débiles en la superficie de los limos y arcillas, pudiendo liberarse por cambios en el pH. Esta observación se corrobora con lo observado en la especiación. Asociaciones fuertes de los metales con la fracción residual e oxihidróxidos de hierro, similares a los determinados en esta investigación han sido reportadas para los sedimentos del Orinoco Medio, Venezuela (4).

Las asociaciones apreciadas en los metales con la granulometría, refleja la compleja mineralogía de la cuenca. La provincia Cuchivero ocupa una porción occidental del Cratón Amazónico, formada por arcos magmáticos de edad 1,98-1,88 Ga que consiste esencialmente de rocas granitoides calcoalcalinas (23). También hay minerales como cuarzo, feldespastos, circón, allanita, biotita allanita, ilmenita, ilmenorutilo, monacita, rutilo, hornblenda y biotita. En esta zona se encuentran yacimientos de bauxita, diamantes, caolín, uranio y columbitatantalita (Coltán) (24).

Los índices de contaminación empleados, los cuales permiten determinar el enriquecimiento del sedimento en relación al metal, el grado de contaminación e incluso la toxicidad del sedimento, indicaron que el factor de enriquecimiento ( $\mathrm{FE}$ ) para $\mathrm{Pb}, \mathrm{FE}=\mathrm{O}$, por lo cual hay deficiencia de enriquecimiento $\mathrm{y} \mathrm{su}$ origen es la roca madre, el valor de $\mathrm{Cr}$ es 3 por lo que hay un enriquecimiento moderado, $\mathrm{Mn}, \mathrm{Zn}$, $\mathrm{Ni}, \mathrm{Co}, \mathrm{Cu}$, están entre 5 y 10 por lo que tienen un enriquecimiento moderado $\mathrm{y}$ 
Cd cuyo valor es de 76, su enriquecimiento es extremadamente alto. Todos los metales excepto $\mathrm{Pb}$, tienen una fuente adicional a la roca madre y no se vislumbra fuentes antropogénicas. Los índices de contaminación (CP) es inferior a 3, el de carga contaminante (ICC) es menor que 8 y el índice de potencial de geoacumulación arrojó valores negativos para todos los metales, lo cual es una señal de que no existe contaminación por metales en los sedimentos (Fig.3E). A pesar de lo indicado por los diferentes índices, el código de evaluación de riesgo ambiental (RAC) indicó que, el riesgo de alteración ambiental que presenta los sedimentos, tanto del río Cuchivero, como del Guaniamo, es bajo para Fe y Co, alto para $\mathrm{Zn}$ y $\mathrm{Ni}$, medio para $\mathrm{Cu}$ y $\mathrm{Cr}$ y muy alto para $\mathrm{Mn}, \mathrm{Cd}$ y $\mathrm{Pb}$, este último metal específicamente para el río Cuchivero (Fig.3F).

\section{Conclusiones}

Los metales $\mathrm{Zn}, \mathrm{Ni}, \mathrm{Pb}$ y $\mathrm{Fe}$ se encuentran asociados preferencialmente a la fracción residual y carbonatos, aunque algunos como $\mathrm{Cu}$, Co y $\mathrm{Cr}$ también pueden presentarse asociados a óxidos de hierro. Metales como, Mn y Cd se encuentran principalmente en la fracción intercambiable. Los índices empleados indican que todos los metales, excepto plomo, tienen como fuente principal de origen la roca madre y algunas que otras fuentes adicionales a la roca madre, no evidenciándose fuentes antropogénicas. No se evidencia enriquecimiento, excepto para cadmio, ni contaminación por ninguno de los metales investigados. El código de evaluación de riesgo ambiental determinó que, el riesgo de alteración ambiental es bajo para $\mathrm{Fe}$ y Co, alto para $\mathrm{Zn}$ y $\mathrm{Ni}$, medio para $\mathrm{Cu}$ y $\mathrm{Cr}$, y muy alto para $\mathrm{Mn}, \mathrm{Cd}$ y $\mathrm{Pb}$.

\section{Agradecimientos}

Los autores agradecen a FUNDACITE GUAYANA toda la colaboración prestada para el desarrollo de esta investigación a través del financiamiento del Proyecto 000606.

\section{Referencias bibliográficas}

1. MORANTES J., YANES C., TOSIANI T., RAMÍREZ A., MARRERO S. Cod Geol Venez. 1998. En: http://www.pdvsa.com/lexico/post ers/jm9801.htm. 13/6/2015.

2. COLVEÉ P., SZEZERBAN E., TALUKDAR S. El rio Orinoco como ecosistema. Weibezahn F., Alvarez H., Lewis Jr W (Eds). Caracas, Venezuela.430 pp.1990.

3. YANES, C. Estudio geoquímico regional de los ríos del Escudo de Guayana (Para obtener el título de Doctor). Facultad de Ciencias y Facultad de Ingeniería, Escuela de Geología, Minas y Geofísica, U.C.V, Venezuela 313 pp. 1997.

4. MÁRQUEZ A., GARCÍA O., SENIOR W MARTÍNEZ G., GONZÁlEZ Á. Ciencias 20 (1): 60-73. 2012.

5. ANDREAE M. The important of chemical speciation. (Eds). Berhand M, Brickman F. y Sadler DK. Springer-Vergag, Berlin (Germany). 301-335. 1986.

6.

http://www.buenastareas.com/ens ayos/Rio-Cuchivero/1749357.html. 13/6/2015 2015.

7. MÁRQUEZ A., SENIOR W., MARTÍNEZ G., GONZÁLEZ Á. Ciencias 17 (1): 87 97. 2009.

8. GONZÁlEZ H., M. RAMIREZ. J Geochem Explore 52 (1-2): 183192.1995.

9. VOGEL A. 1960. Química Analítica Cuantitativa. Vol. 1. (Eds). Kapelusz, S.A Buenos Aires (Argentina). 811 pp. 1995. 
10. TESSIER A., CAMPBELL P., BISSON M. Anal Chem 45: 844851.1979

11. RUBIO, B., NOMBELA M. A., VILAS F. Mar Poll Bull 40: 11. 968-980. 2000

12. SUBRAMANIAN V., \& G. MOHANACHANDRAN. Mar Poll Bull 2: 324-330. 1990.

13. SAHU K C., BHOSALE U. Chemi Geol 91: 263-83.1991.

14. TUREKIANK., WEDEPOHL K. Geol Soc of Amer Bull 72:175192. 1961.

15. MÜLLER G. Umschan. 79: 778783. 1979

16. COVELLI S., FONTOLAN G.. Envir Geol 30: 34-45.1997

17. APRILE F., BOUVY M. Braz $\boldsymbol{J}$ of Aquatic Sci Tech 12(1):1-8. 2008.

18. TAYLOR S. Geoch Cosmochem Acta 28:1273-1285. 1964.

19 LAWSON D. \&. WINCHESTER J. Atmosph Envir.10:925-930.1979.

20. DAVAULTER, V. \& S. ROGNERUD. 2001. Chemosphere. 42:9-18.

21.TOMLINSON D., WILSON J., HARRIS C., JEFFNEY D. Helgol Wiss Meeresunters. 33: 566572. 1980.

22. PERIN G., CRABOLEDDA L., LÚCHESE M., CIRILLO R., DOTTA L., ZANETTA M. L., ORO A. A. Heavy metal speciation in the sediments of northern Adriatic Sea. A new approach for environmental toxicity determination. In: Lakkas, T. D. (Ed), Heavy Metals in the Environment, 2. 1985

23. TASSINARI G, BETTENCOURT S., GERALDES C., MACAMBRIA B., LAFON M.The Amazonian Craton. En: Tectonic Evolution of South America. Brazil Pub Esp. 31. Int Geol Cong. 41-95pp. 2000

24. GAMERO, A, M. REVETI. R. JIMÉNEZ, Y. RODRÍGUEZ, F. ANGULO, R. HURTADO, A. ABRISQUETA. Rev Fac Ing $\boldsymbol{U} \boldsymbol{C}$ V 29(4): 25-40. 2014. 


\section{$(\mathrm{C}][\mathrm{E} \mathbb{N}(\mathrm{C}][\mathrm{A}$ \\ Revista Científica de la Facultad Experimental de Ciencias de la Universidad del Zulia \\ Vol. $24 \mathrm{~N}^{\mathbf{0}} 3$, Julio - Septiembre 2016}

Pg.

\section{BIOLOGÍA/BIOLOGY}

Adquisición de la resistencia a cromo en E. coli $\mathrm{DH}_{5}$ a por transformación in vitro mediada por plásmidos de Acinetobacter sp.

Acquisition of the chromium resistance in $E$. coli DH5a through in vitro transformation mediated by Acinetobacter sp plasmids

Jesús Pérez, Doris Reyes, Arnaldo Armado y Oscar Valbuena

(Valencia, Venezuela)

\section{QUÍMICA/ CHEMISTRY}

Formación de complejos ternarios entre el sistema Niquel(II)-Ácido Dipicolínico y algunos ligandos bidentados

Ternary complexes formation between the Nickel(II)-Dipicolinic acid system and some bidentate ligands

Isaac Barrera, Mary Lorena Araujo, Felipe Brito, Alejandro Pérez, Lino Hernández, Edgar Del Carpio y Vito Lubes

(Caracas, Venezuela)

Especiación de metales en sedimentos del río Cuchivero, Venezuela Speciation of heavy metals in sediments of the Cuchivero River, Venezuela Aristide Márquez, Gregorio Martínez, Julio Figuera, William Senior, Antonio Benítez, Ángel González

(Sucre, Venezuela)

Authentication of the protected designation of origin "Kaki Ribera del Xúquer" from its mineral profile Autenticación de la denominación de origen protegida "Kaki Ribera del Xúquer" a partir de su perfil mineral

Alba Mir-Marqués, Maria Luisa Cervera, Miguel de la Guardia (Valencia, Spain) 